Mathematical Modelling and Analysis

Volume 16 Number 2, June 2011, 260-272

Doi:10.3846/13926292.2011.580015

(C) Vilnius Gediminas Technical University, 2011
www.tandf.co.uk/journals/TMMA

Publisher: Taylor\&Francis and VGTU

Online ISSN: 1648-3510

Print ISSN: 1392-6292

\title{
On Approximation of Value Functions for Controlled Discontinuous Random Processes
}

\author{
Svetlana Danilenko ${ }^{a}$ and Henrikas Pragarauskas ${ }^{b}$ \\ ${ }^{a}$ Vilnius Gediminas Technical University \\ Sauletekio al. 11, LT-10223 Vilnius, Lithuania \\ ${ }^{b}$ Institute of Mathematics and Informatics, Vilnius University \\ Akademijos 4, LT-08663 Vilnius, Lithuania \\ E-mail: svetlana.danilenko@vgtu.lt \\ E-mail(corresp.): pragarauskash@yahoo.com
}

Received August 31, 2010; revised January 13, 2011; published online May 1, 2011

\begin{abstract}
We consider the problem of approximation of value functions for controlled possibly degenerated diffusion processes with jumps by using piece-wise constant control policies. A rate of convergence for the corresponding value functions is established provided that the coefficients of controlled processes are sufficiently smooth. The paper extends the results of N.V. Krylov to a more general class of controlled processes.
\end{abstract}

Keywords: Controlled diffusion processes with jumps, Bellman principle, piece-wise constant strategies, value functions.

AMS Subject Classification: $60 \mathrm{~J} 75$.

\section{Introduction}

The paper is devoted to numerical approximations in the control theory of random processes. Recall that under suitable assumptions such value functions are probabilistic solutions to fully nonlinear parabolic integro-differential Bellman equations of the second order (see [3]). In the paper, the analysis of approximations of value functions is based on the same ideas as that for value functions of controlled diffusion processes considered by N.V. Krylov in [2].

The paper is organised as follows. The main results are given in Section 2. Section 3 contains auxiliary results. In Section 4 , the proofs of the main results are presented.

Throughout the paper $R^{d}$ is a $d$-dimensional Euclidean space, $A$ is a separable metric space, $T \in(0, \infty), K \in[1, \infty), \delta_{0} \in(0,1]$ and $\delta \in(0,1]$ are some fixed constants. By $N$ we denote various constants depending only on $T, K, d$ and $d_{1}$, where $d_{1}$ is introduced in the next section. 


\section{Main Results}

Let $\Pi$ be a $\sigma$-finite nonnegative measure on $\left(R^{m}, \mathcal{B}\left(R^{m}\right)\right)$ such that $\Pi(z:|z|>$ $\varepsilon)<\infty$ for any $\varepsilon>0$. Let $(\Omega, \mathcal{F}, \mathbf{P})$ be a complete probability space with a filtration of $\sigma$-algebras $\mathbb{F}=\left(\mathcal{F}_{t}, t \geq 0\right)$ satisfying the usual conditions. Assume that on this probability space a $d_{1}$-dimensional $\mathbb{F}$-adapted Wiener process $W_{t}, t \geq 0$, and $\mathbb{F}$-adapted Poisson random measure $p(d t, d z)$ on $([0, \infty) \times$ $\left.R^{m}, \mathcal{B}([0, \infty)) \otimes \mathcal{B}\left(R^{m}\right)\right)$ with a compensator $\Pi(d z) d t$ are given. Let

$$
q(d t, d z)=p(d t, d z)-\Pi(d z) d t
$$

be a martingale measure.

Definition 1. An $A$-valued random process $\alpha_{t}=\alpha_{t}(\omega), t \geq 0, \omega \in \Omega$, is called $\mathbb{F}$-admissible if it is $\mathcal{F} \otimes \mathcal{B}([0, \infty))$-measurable and $\alpha_{t}$ is $\mathcal{F}_{t}$-measurable for each $t \geq 0$.

The set of all $\mathbb{F}$-admissible processes is denoted by $\mathfrak{A}$. Let $\mathfrak{A}_{h}$ be the subset of $\mathfrak{A}$ consisting of all processes $\alpha_{t}$ which are constant on intervals $\left[0, h^{2}\right)$, $\left[h^{2}, 2 h^{2}\right)$, etc.

Fix integers $d \geq 1, d_{1} \geq 1, m \geq 1$ and assume that we have the following functions: a $d \times d_{1}$ matrix-valued $\sigma(\alpha, t, x)$ and $R^{d}$-valued $b(\alpha, t, x)$ on $A \times$ $[0, \infty) \times R^{d}$, a $R^{d}$-valued $c(\alpha, t, x, z)$ on $A \times[0, \infty) \times R^{d} \times R^{m}$ and real-valued $g(x)$ on $R^{d}$. We assume that these functions are Borel measurable.

For any matrix $\sigma=\left(\sigma_{i j}\right)$ and function $c: R^{m} \rightarrow R^{d}$ denote

$$
\|\sigma\|=\left\{\sum_{i, j} \sigma_{i j}^{2}\right\}^{1 / 2}, \quad\|c\|_{2, \Pi}=\left\{\int_{R^{m}}|c(z)|^{2} \Pi(d z)\right\}^{1 / 2} .
$$

Further we shall use the following assumption.

Assumption 1. (i) The functions $\sigma$ and $b$ are continuous with respect to $\alpha$ and for each $\alpha \in A, t \in[0, \infty), x \in R^{d}$ we have

$$
\left\|c\left(\alpha_{n}, t, x, \cdot\right)-c(\alpha, t, x, \cdot)\right\|_{2, \Pi} \rightarrow 0 \text { as } \alpha_{n} \rightarrow \alpha ;
$$

(ii) For each $\alpha \in A, t \in[0, \infty), x, y \in R^{d}$

$$
\begin{aligned}
& \|\sigma(\alpha, t, x)\|+|b(\alpha, t, x)|+\|c(\alpha, t, x, \cdot)\|_{2, \Pi} \leq K, \\
& \begin{aligned}
\|\sigma(\alpha, t, x)-\sigma(\alpha, t, y)\|+|b(\alpha, t, x)-b(\alpha, t, y)| \\
\quad+\|c(\alpha, t, x, \cdot)-c(\alpha, t, y, \cdot)\|_{2, \Pi} \leq K|x-y|
\end{aligned}
\end{aligned}
$$

(iii) For each $\alpha \in A, s, t \in[0, \infty), x \in R^{d}$

$$
\begin{aligned}
\| \sigma(\alpha, t, x) & -\sigma(\alpha, s, x) \|+|b(\alpha, t, x)-b(\alpha, s, x)| \\
& +\|c(\alpha, t, x, \cdot)-c(\alpha, s, x, \cdot)\|_{2, \Pi} \leq K|t-s|^{\delta_{0} / 2}
\end{aligned}
$$

(iv) For each $x, y \in R^{d}$

$$
|g(x)| \leq K, \quad|g(x)-g(y)| \leq K|x-y|^{\delta} .
$$


Let Assumption 1 (ii) be satisfied. Then, by Itô's theorem, for each $\alpha \in \mathfrak{A}$, $s \in[0, T]$ and $x \in R^{d}$ there exists a unique solution $X_{t}=X_{t}^{\alpha, s, x}, t \geq 0$, of the equation

$$
\begin{aligned}
X_{t}=x & +\int_{0}^{t} \sigma\left(\alpha_{r}, s+r, X_{r}\right) d W_{r} \\
& +\int_{0}^{t} b\left(\alpha_{r}, s+r, X_{r}\right) d r+\int_{0}^{t} \int c\left(\alpha_{r}, s+r, X_{r}, z\right) q(d r, d z) .
\end{aligned}
$$

For $\alpha \in \mathfrak{A}, s \in[0, T]$ and $x \in R^{d}$ define

$$
v^{\alpha}(s, x)=\mathbb{E} g\left(X_{T-s}^{\alpha, s, x}\right), \quad v(s, x)=\sup _{\alpha \in \mathfrak{A}} v^{\alpha}(s, x), \quad v_{h}(s, x)=\sup _{\alpha \in \mathfrak{A}_{h}} v^{\alpha}(s, x) .
$$

Theorem 1. Let Assumption 1 be satisfied. Then for each $s \in[0, T], x \in R^{d}$, $h \in(0,1)$

$$
\left|v(s, x)-v_{h}(s, x)\right| \leq N h^{\varkappa},
$$

where $\varkappa=\delta \delta_{0}^{2} /\left(2+\delta \delta_{0}+\delta_{0}-\delta\right)$ and the constant $N$ depends only on $T, K, d$ and $d_{1}$.

Remark 1. The largest value of $\varkappa$ for $\delta_{0} \in(0,1], \delta \in(0,1]$ is equal to $1 / 3$ and is achieved with $\delta_{0}=\delta=1$.

Theorem 1 can be used to reduce the problem of calculation of value function $v$ to that of value function $v_{h}$ for piece-wise constant control policies.

For $\alpha \in A, 0 \leq s \leq t<\infty, x \in R^{d}$ and any Borel measurable bounded real-valued function $f$ on $R^{d}$ define

$$
G_{s, t}^{\alpha} f(x)=\mathbb{E} f\left(X_{t-s}^{\alpha, s, x}\right), \quad G_{s, t} f(x)=\sup _{\alpha \in A} G_{s, t}^{\alpha} f(x) .
$$

According to the dynamic programming principle (see Lemma 1 in the next section) for $s+h^{2} \leq T$

$$
v_{h}(s, x)=G_{s, s+h^{2}} v_{h}\left(s+h^{2}, \cdot\right)(x) .
$$

Therefore $v_{h}(s, x)$ can be found from its boundary value $v_{h}(T, \cdot)=g(\cdot)$ by backward iteration.

In order to simplify the calculation one can apply the Euler scheme as the simplest approximation of controlled process. For $\alpha \in A, s, t \geq 0, x \in R^{d}$ define

$$
Y_{t}^{\alpha, s, x}=x+\sigma(\alpha, s, x) W_{t}+b(\alpha, s, x) t+\int_{0}^{t} \int c(\alpha, s, x, z) q(d r, d z)
$$

and recursively

$$
\begin{aligned}
& \bar{v}_{h}(s, x)=g(x) \quad \text { if } \quad s \in\left(T-h^{2}, T\right], \\
& \bar{v}_{h}(s, x)=\bar{G}_{s, s+h^{2}} \bar{v}_{h}\left(s+h^{2}, \cdot\right)(x) \quad \text { if } s \leq T-h^{2},
\end{aligned}
$$

where

$$
\bar{G}_{s, s+t}^{\alpha} f(x)=\mathbb{E} f\left(Y_{t}^{\alpha, s, x}\right), \quad \bar{G}_{s, s+t} f(x)=\sup _{\alpha \in A} \bar{G}_{s, s+t}^{\alpha} f(x) .
$$


Theorem 2. Let Assumption 1 be satisfied. Then for each $s \in[0, T], x \in R^{d}$ and $h \in(0,1)$

$$
\left|v(s, x)-\bar{v}_{h}(s, x)\right| \leq N h^{\varkappa},
$$

$\varkappa=\delta \delta_{0}^{2} /\left(2+\delta \delta_{0}+\delta_{0}-\delta\right)$ and the constant $N$ depends only on $T, K, d$ and $d_{1}$.

\section{Auxiliary Results}

The following lemma states the dynamic programming principle for the value functions $v$ and $v_{h}$.

Lemma 1. Let Assumption 1 be satisfied. Then:

(i) for each $x \in R^{d}$ and $0 \leq s \leq t \leq T$

$$
v(s, x)=\sup _{\alpha \in \mathfrak{A}} \mathbb{E} v\left(t, X_{t-s}^{\alpha, s, x}\right)
$$

(ii) for each $x \in R^{d}$ and $0 \leq s \leq t \leq T$ such that $(t-s) / h^{2}$ is an integer

$$
v_{h}(s, x)=\sup _{\alpha \in \mathfrak{A}_{h}} \mathbb{E} v_{h}\left(t, X_{t-s}^{\alpha, s, x}\right) .
$$

The proof of the lemma is similar to that for controlled diffusion processes (see Theorem 3.1.6, Exercise 3.2.1 and Lemma 3.3.1 in [1]) and therefore it is omitted here.

In order to prove the main results of the paper, we introduce an auxiliary controlled process and value functions $u$ and $u_{h}$ as follows. Let

$$
B_{1}=\left\{x \in R^{d}:|x|<1\right\}, \quad B=A \times\left\{(r, \xi): r \in(-1,0), \xi \in B_{1}\right\} .
$$

We extend the functions $\sigma, b, c$ for $t<0$ by

$$
\sigma(\alpha, t, x)=\sigma(\alpha, 0, x), \quad b(\alpha, t, x)=b(\alpha, 0, x), \quad c(\alpha, t, x, z)=c(\alpha, 0, x, z)
$$

and for a fixed $\varepsilon \in(0,1)$ and each $\beta=(\alpha, r, \xi) \in B, t \in R, x \in R^{d}, z \in R^{m}$ define

$$
\begin{aligned}
& \sigma(\beta, t, x)=\sigma\left(\alpha, t+\varepsilon^{2} r, x+\varepsilon \xi\right), \quad b(\beta, t, x)=b\left(\alpha, t+\varepsilon^{2} r, x+\varepsilon \xi\right), \\
& c(\beta, t, x, z)=c\left(\alpha, t+\varepsilon^{2} r, x+\varepsilon \xi, z\right) .
\end{aligned}
$$

Let $\mathfrak{B}$ be the set of all measurable $\mathbb{F}$-adapted $B$-valued processes and $\mathbb{B}_{h}$ be the subset of $\mathfrak{B}$ consisting of functions which are constant on intervals $\left[0, h^{2}\right)$, $\left[h^{2}, 2 h^{2}\right)$, etc. Finally, for each $\beta \in \mathfrak{B}, s \in[0, S], x \in R^{d}$, we define a controlled jump-diffusion process $X_{t}^{\beta, s, x}, t \geq 0$, as a solution to (2.1) with $\alpha_{r}$ replaced by $\beta_{r}$ and the value functions

$$
u^{\beta}(s, x)=\mathbb{E} g\left(X_{S-s}^{\beta, s, x}\right), u(s, x)=\sup _{\beta \in \mathfrak{B}} u^{\beta}(s, x), u_{h}(s, x)=\sup _{\beta \in \mathfrak{B}_{h}} u^{\beta}(s, x),
$$

where $S=T+\varepsilon^{2}$. Obviously, the process $X_{t}^{\beta, s, x}$ and value functions $u, u_{h}$ depend also on $\varepsilon$ what is not explicitly shown just for brevity of notation. 
Remark 2. Since the controlled process $X_{t}^{\beta, s, x}$ and the value functions $u, u_{h}$ are defined in the same way as $X_{t}^{\alpha, s, x}, v$ and $v_{h}$, Lemma 1 implies that for each $x \in R^{d}$ and $0 \leq s \leq t \leq S$

$$
u(s, x)=\sup _{\beta \in \mathfrak{B}} \mathbb{E} u\left(t, X_{t-s}^{\beta, s, x}\right)
$$

and for each $x \in R^{d}$ and $0 \leq s \leq t \leq S$ such that $(t-s) / h^{2}$ is an integer

$$
u_{h}(s, x)=\sup _{\beta \in \mathfrak{B}_{h}} \mathbb{E} u_{h}\left(t, X_{t-s}^{\beta, s, x}\right) .
$$

Let $y_{t}^{(i)}, t \geq 0, i=1,2$, be $d$-dimensional solutions to the equations $y_{t}=x^{(i)}+\int_{0}^{t} \sigma^{(i)}\left(s, y_{s}\right) d W_{s}+\int_{0}^{t} b^{(i)}\left(s, y_{s}\right) d s+\int_{0}^{t} \int c^{(i)}\left(s, y_{s}, z\right) q(d s, d z)$, respectively, the coefficients of which are measurable and $\mathbb{F}$-adapted random functions satisfying the linear growth and Lipschitz conditions, i.e. for each $t \geq 0, x, y \in R^{d}, \omega \in \Omega$ and $i=1,2$

$$
\begin{gathered}
\left\|\sigma^{(i)}(t, x)\right\|+\left|b^{(i)}(t, x)\right|+\left\|c^{(i)}(t, x, \cdot)\right\|_{2, \Pi} \leq K(1+|x|) \\
\left\|\sigma^{(i)}(t, x)-\sigma^{(i)}(t, y)\right\|+\left|b^{(i)}(t, x)-b^{(i)}(t, y)\right| \\
\left.+\left\|c^{(i)}(t, x, \cdot)-c^{(i)}(t, y, \cdot)\right\|_{2, \Pi} \leq K|x-y|\right) .
\end{gathered}
$$

Lemma 2. Let the above assumptions be satisfied and $T \in(0, \infty)$. Then there is a constant $N=N(K, T)$ such that

$$
\begin{aligned}
& \mathbb{E} \sup _{t \leq T}\left|y_{t}^{(1)}-y_{t}^{(2)}\right|^{2} \leq N\left(\left|x^{(1)}-x^{(2)}\right|^{2}+\mathbb{E} \int_{0}^{T}\left(\left\|\sigma^{(1)}\left(s, y_{s}^{(2)}\right)-\sigma^{(2)}\left(s, y_{s}^{(2)}\right)\right\|\right.\right. \\
& \left.\quad+\left|b^{(1)}\left(s, y_{s}^{(2)}\right)-b^{(2)}\left(s, y_{s}^{(2)}\right)\right|^{2}+\left\|c^{(1)}\left(s, y_{s}^{(2)}, \cdot\right)-c^{(2)}\left(s, y_{s}^{(2)}, \cdot\right)\right\|_{2, \Pi}^{2}\right) d s
\end{aligned}
$$

Proof. Using well-known properties of stochastic integrals, we have

$$
\begin{aligned}
& \mathbb{E} \sup _{t \leq T}\left|y_{t}^{(1)}-y_{t}^{(2)}\right|^{2} \leq N\left(\left|x^{(1)}-x^{(2)}\right|^{2}+\mathbb{E} \int_{0}^{T}\left(\left\|\sigma^{(1)}\left(s, y_{s}^{(1)}\right)-\sigma^{(2)}\left(s, y_{s}^{(2)}\right)\right\|^{2}\right.\right. \\
& \left.\quad+\left|b^{(1)}\left(s, y_{s}^{(1)}\right)-b^{(2)}\left(s, y_{s}^{(2)}\right)\right|^{2}+\left\|c^{(1)}\left(s, y_{s}^{(1)}, \cdot\right)-c^{(2)}\left(s, y_{s}^{(2)}, \cdot\right)\right\|_{2, \Pi}^{2}\right) d s,
\end{aligned}
$$

where the constant $N$ depends only on $K$ and $T$. According to our assumptions,

$$
\left\|\sigma^{(1)}\left(s, y_{s}^{(1)}\right)-\sigma^{(2)}\left(s, y_{s}^{(2)}\right)\right\| \leq K\left|y_{s}^{(1)}-y_{s}^{(2)}\right|+\left\|\sigma^{(1)}\left(s, y_{s}^{(2)}\right)-\sigma^{(2)}\left(s, y_{s}^{(2)}\right)\right\|
$$

and similar inequalities hold for other terms on the right-hand side of (3.1). Therefore, the assertion of the lemma follows by Gronwall's inequality.

Lemma 3. Let Assumption 1 be satisfied, $s, s^{\prime} \in[0, S], x, y \in R^{d}$ and $\beta=$ $(\alpha, r, \xi) \in \mathfrak{B}$. Then there is a constant $N=N(T, K)$ such that

$$
\begin{aligned}
& \mathbb{E} \sup _{t \leq S}\left|X_{t}^{\beta, s, x}-X_{t}^{\alpha, s, x}\right|^{2} \leq N \varepsilon^{2 \delta_{0}}, \quad \mathbb{E} \sup _{t \leq S}\left|X_{t}^{\beta, s, x}-X_{t}^{\beta, s, y}\right|^{2} \leq N|x-y|^{2}, \\
& \mathbb{E} \sup _{t \leq S}\left|X_{t}^{\beta, s, x}-X_{t}^{\beta, s^{\prime}, x}\right|^{2} \leq N\left|s-s^{\prime}\right|^{\delta_{0}},
\end{aligned}
$$


Proof. The estimates of the lemma follow easily from Lemma 2 and Assumption 1. For example,

$$
\begin{aligned}
\mathbb{E} \sup _{t \leq S}\left|X_{t}^{\beta, s, x}-X_{t}^{\alpha, s, x}\right|^{2} & \leq N \sup \left(\left\|\sigma\left(\alpha, t+\varepsilon^{2} r, x+\varepsilon \xi\right)-\sigma(\alpha, t, x)\right\|^{2}\right. \\
& +\left|b\left(\alpha, t+\varepsilon^{2} r, x+\varepsilon \xi\right)-b(\alpha, t, x)\right|^{2} \\
& \left.+\left\|c\left(\alpha, t+\varepsilon^{2} r, x+\varepsilon \xi, \cdot\right)-c(\alpha, t, x, \cdot)\right\|_{2, \Pi}^{2}\right) \leq N \varepsilon^{2 \delta_{0}}
\end{aligned}
$$

where $N=N(T, K)$ and sup is taken over $\alpha \in A, t \leq S, r \in(-1,0), x \in R^{d}$, $\xi \in B_{1}$.

Remark 3. Lemma 2 and Hölder's inequality imply that for each $\alpha \in \mathfrak{A}, s, t \geq 0$, $x \in R^{d}$ and $\mu \in(0,2]$

$$
\mathbb{E}\left|X_{t}^{\alpha, s, x}-x\right|^{\mu} \leq\left\{\mathbb{E} X_{t}^{\alpha, s, x}-\left.x\right|^{2}\right\}^{\frac{\mu}{2}} \leq N t^{\frac{\mu}{2}},
$$

where the constant $N=N(K)$.

Lemma 4. Let Assumption 1 be satisfied. Then for each $s \in[0, T], x \in R^{d}$ and $\beta=(\alpha, r, \xi) \in \mathfrak{B}$

$$
\begin{aligned}
& \left|u^{\beta}(s, x)-v^{\alpha}(s, x)\right| \leq N \varepsilon^{\delta_{0} \delta}, \quad|u(s, x)-v(s, x)| \leq N \varepsilon^{\delta_{0} \delta} \\
& \left|u_{h}(s, x)-v_{h}(s, x)\right| \leq N \varepsilon^{\delta_{0} \delta}
\end{aligned}
$$

where the constant $N=N(T, K)$.

Proof. As can be easily seen, it suffices to prove the first inequality. By Assumption 1, Hölder's inequality and Lemma 3,

$$
\begin{aligned}
\left|u^{\beta}(s, x)-v^{\alpha}(s, x)\right| & \leq \mathbb{E}\left|g\left(X_{S-s}^{\beta, s, x}\right)-g\left(X_{T-s}^{\alpha, s, x}\right)\right| \\
& \leq K \mathbb{E}\left|X_{S-s}^{\beta, s, x}-X_{T-s}^{\alpha, s, x}\right|^{\delta} \leq K\left\{\mathbb{E}\left|X_{S-s}^{\beta, s, x}-X_{T-s}^{\alpha, s, x}\right|^{2}\right\}^{\delta / 2} \\
& \leq 2 K\left\{\mathbb{E}\left|X_{S-s}^{\beta, s, x}-X_{T-s}^{\beta, s, x}\right|^{2}+\mathbb{E}\left|X_{T-s}^{\beta, s, x}-X_{T-s}^{\alpha, s, x}\right|^{2}\right\}^{\delta / 2} \\
& \leq N\left(\varepsilon^{2}+\varepsilon^{2 \delta_{0}}\right)^{\delta / 2} \leq N \varepsilon^{\delta_{0} \delta} .
\end{aligned}
$$

Lemma 5. Let Assumption 1 be satisfied. Then there is a constant $N=$ $N(T, K)$ such that:

(i) for each $s \in[0, S]$ and $x, y \in R^{d}$

$$
|u(s, x)-u(s, y)| \leq N|x-y|^{\delta}, \quad\left|u_{h}(s, x)-u_{h}(s, y)\right| \leq N|x-y|^{\delta} ;
$$

(ii) for each $0 \leq s \leq t \leq S$ and $x \in R^{d}$

$$
|u(s, x)-u(t, x)| \leq N|t-s|^{\delta / 2}, \quad\left|u_{h}(s, x)-u(t, x)\right| \leq N\left(h^{\delta_{0} \delta}+|t-s|^{\delta / 2}\right) .
$$

Proof. (i) By Assumption 1, Hölder's inequality and Lemma 3,

$$
\begin{aligned}
|u(s, x)-u(s, y)| & \leq \sup _{\beta \in \mathfrak{B}} \mathbb{E}\left|g\left(X_{S-s}^{\beta, s, x}\right)-g\left(X_{S-s}^{\beta, s, y}\right)\right| \leq K \sup _{\beta \in \mathfrak{B}} \mathbb{E}\left|X_{S-s}^{\beta, s, x}-X_{S-s}^{\beta, s, y}\right|^{\delta} \\
& \leq K \sup _{\beta \in \mathfrak{B}}\left\{\mathbb{E}\left|X_{S-s}^{\beta, s, x}-X_{S-s}^{\beta, s, y}\right|^{2}\right\}^{\delta / 2} \leq N|x-y|^{\delta} .
\end{aligned}
$$


The same arguments prove the second assertion in (i).

(ii) By Remark 2, the assertion (i), Hölder's inequality and Lemma 2,

$$
\begin{aligned}
|u(s, x)-u(t, x)| & =\left|\sup _{\beta \in \mathfrak{B}} \mathbb{E} u\left(t, X_{t-s}^{\beta, s, x}\right)-u(t, x)\right| \leq N \sup _{\beta \in \mathfrak{B}} \mathbb{E}\left|X_{t-s}^{\beta, s, x}-x\right|^{\delta} \\
& \leq N \sup _{\beta \in \mathfrak{B}}\left\{\mathbb{E}\left|X_{t-s}^{\beta, s, x}-x\right|^{2}\right\}^{\delta / 2} \leq N|t-s|^{\delta / 2} .
\end{aligned}
$$

Similarly, if $(t-s) / h^{2}$ is an integer,

$$
\left|u_{h}(s, x)-u_{h}(t, x)\right| \leq N|t-s|^{\delta / 2} .
$$

On the other hand, by Hölder's inequality and Lemmas 2, 3, for each $0 \leq$ $s \leq t \leq S$ and $x \in R^{d}$

$$
\begin{aligned}
& \left|u_{h}(s, x)-u_{h}(t, x)\right| \leq \sup _{\beta \in \mathfrak{B}_{h}} \mathbb{E}\left|g\left(X_{S-s}^{\beta, s, x}\right)-g\left(X_{S-t}^{\beta, t, x}\right)\right| \\
& \quad \leq K \sup _{\beta \in \mathfrak{B}_{h}} \mathbb{E}\left|X_{S-s}^{\beta, s, x}-X_{S-t}^{\beta, t, x}\right|^{\delta} \leq K \sup _{\beta \in \mathfrak{B}_{h}}\left\{\mathbb{E}\left|X_{S-s}^{\beta, s, x}-X_{S-t}^{\beta, t, x}\right|^{2}\right\}^{\delta / 2} \\
& \quad \leq 2 K \sup _{\beta \in \mathfrak{B}_{h}}\left\{\mathbb{E}\left|X_{S-s}^{\beta, s, x}-X_{S-t}^{\beta, s, x}\right|^{2}+\mathbb{E}\left|X_{S-t}^{\beta, s, x}-X_{S-t}^{\beta, t, y}\right|^{2}\right\}^{\delta / 2} \\
& \quad \leq N\left(|t-s|+|t-s|^{\delta_{0}}\right)^{\delta / 2} \leq N|t-s|^{\delta_{0} \delta / 2} .
\end{aligned}
$$

The inequalities (3.2) and (3.3) imply the second assertion in (ii). The lemma is proved.

For $\alpha \in A$ and smooth functions $f:[0, \infty) \times R^{d} \rightarrow R$ introduce the operator

$$
\begin{aligned}
L^{\alpha} f(t, x)= & \frac{\partial f}{\partial t}(t, x)+\sum_{i, j=1}^{d} a_{i j}(\alpha, t, x) \frac{\partial^{2} f}{\partial x_{i} \partial x_{j}}(t, x) \\
& +\sum_{i=1}^{d} b_{i}(\alpha, t, x) \frac{\partial f}{\partial x_{i}}(t, x)+\int_{R^{m}} \nabla_{c(\alpha, t, x, z)}^{2} f(t, x) \Pi(d z),
\end{aligned}
$$

where $a=\frac{1}{2} \sigma \sigma^{*}$ and

$$
\nabla_{y}^{2} f(t, x)=f(t, x+y)-f(t, x)-\sum_{i=1}^{d} \frac{\partial f}{\partial x_{i}}(t, x) y_{i} .
$$

Let a nonnegative function $\zeta \in C_{0}^{\infty}\left((-1,0) \times B_{1}\right)$ be such that

$$
\int_{-1}^{0} \int_{B_{1}} \zeta(t, x) d t d x=1 .
$$

For $\varepsilon>0$ define $\zeta_{\varepsilon}(t, x)=\varepsilon^{-d-2} \zeta\left(t / \varepsilon^{2}, x / \varepsilon\right)$. Further we use the following notation:

$$
\begin{aligned}
& f^{(\varepsilon)}(t, x)=\int_{R^{d+1}} \zeta_{\varepsilon}(t-s, x-y) f(s, y) d s d y,|f|_{0}=\sup _{\substack{t \in[0, T] \\
x \in R^{d}}}|f(t, x)|, \\
& {[f]_{\frac{\varkappa}{2}, \varkappa}=\sup _{\substack{s, t \in[0, T] \\
x, y \in R^{d}}} \frac{|f(s, x)-f(t, y)|}{|s-t|^{\varkappa / 2}+|x-y|^{\varkappa}}, \quad \varkappa \in(0,1] .}
\end{aligned}
$$


Lemma 6. Let Assumption 1 be satisfied and $\varepsilon \geq h^{\delta_{0}}$. Then for each $\alpha \in A$

$$
\left[L^{\alpha} u_{h}^{(\varepsilon)}\right]_{\frac{\delta_{0}}{2}, \delta_{0}} \leq N \varepsilon^{-2-\delta_{0}+\delta}
$$

where the constant $N=N\left(T, K, d, d_{1}\right)$.

Proof. Using Lemma 5 and the inequality $\varepsilon \geq h^{\delta_{0}}$, we have for $s \in[0, T]$ and $x \in R^{d}$

$$
\begin{aligned}
\left|\frac{\partial}{\partial s} u_{h}^{(\varepsilon)}(s, x)\right| & =\varepsilon^{-2}\left|\int_{R^{d+1}} u_{h}\left(s-\varepsilon^{2} r, x-\varepsilon \xi\right) \frac{\partial}{\partial s} \zeta(r, \xi) d r d \xi\right| \\
& =\varepsilon^{-2}\left|\int_{R^{d+1}}\left[u_{h}\left(s-\varepsilon^{2} r, x-\varepsilon \xi\right)-u_{h}(s, x)\right] \frac{\partial}{\partial s} \zeta(r, \xi) d r d \xi\right| \\
& \leq N \varepsilon^{-2}\left(h^{\delta \delta_{0}}+\varepsilon^{\delta}\right) \leq N \varepsilon^{-2+\delta}
\end{aligned}
$$

and

$$
\begin{aligned}
\left|\frac{\partial}{\partial x_{i}} u_{h}^{(\varepsilon)}(s, x)\right| & =\varepsilon^{-1}\left|\int_{R^{d+1}} u_{h}\left(s-\varepsilon^{2} r, x-\varepsilon \xi\right) \frac{\partial}{\partial x_{i}} \zeta(r, \xi) d r d \xi\right| \\
& =\varepsilon^{-1}\left|\int_{R^{d+1}}\left[u_{h}\left(s-\varepsilon^{2} r, x-\varepsilon \xi\right)-u_{h}(s, x)\right] \frac{\partial}{\partial x_{i}} \zeta(r, \xi) d r d \xi\right| \\
& \leq N \varepsilon^{-1}\left(h^{\delta \delta_{0}}+\varepsilon^{\delta}\right) \leq N \varepsilon^{-1+\delta} .
\end{aligned}
$$

Similarly,

$$
\begin{aligned}
& \left|\frac{\partial^{2}}{\partial x_{i} \partial x_{j}} u_{h}^{(\varepsilon)}\right|_{0} \leq N \varepsilon^{-2+\delta}, \\
& \left|\frac{\partial^{2}}{\partial s \partial x_{i}} u_{h}^{(\varepsilon)}\right|_{0}+\left|\frac{\partial^{3}}{\partial x_{i} \partial x_{j} \partial x_{k}} u_{h}^{(\varepsilon)}\right|_{0} \leq N \varepsilon^{-3+\delta}, \\
& \left|\frac{\partial^{2}}{\partial s^{2}} u_{h}^{(\varepsilon)}\right|_{0}+\left|\frac{\partial^{3}}{\partial s \partial x_{i} \partial x_{j}} u_{h}^{(\varepsilon)}\right|_{0} \leq N \varepsilon^{-4+\delta} .
\end{aligned}
$$

Let us prove the inequality

$$
\left[\frac{\partial}{\partial s} u_{h}^{(\varepsilon)}\right]_{\frac{\delta_{0}}{2}, \delta_{0}} \leq N \varepsilon^{-2-\delta_{0}+\delta} .
$$

Assume that $|t-s|^{\frac{1}{2}}+|x-y| \leq \varepsilon$. Then using (3.7) and (3.8) we have

$$
\begin{aligned}
\left|\frac{\partial}{\partial s} u_{h}^{(\varepsilon)}(s, x)-\frac{\partial}{\partial s} u_{h}^{(\varepsilon)}(t, y)\right| & \leq|t-s|\left|\frac{\partial^{2}}{\partial s^{2}} u_{h}^{(\varepsilon)}\right|_{0}+N|x-y|\left|\frac{\partial^{2}}{\partial s \partial x_{i}} u_{h}^{(\varepsilon)}\right|_{0} \\
& \leq N\left(|t-s| \varepsilon^{-4+\delta}+|x-y| \varepsilon^{-3+\delta}\right) \\
& \leq N \varepsilon^{-2-\delta_{0}+\delta}\left(|t-s|^{\frac{\delta_{0}}{2}}+|x-y|^{\delta_{0}}\right) .
\end{aligned}
$$

Assume that $|t-s|^{\frac{1}{2}}+|x-y| \geq \varepsilon$. Then using (3.4) we have

$$
\begin{aligned}
\left|\frac{\partial}{\partial s} u_{h}^{(\varepsilon)}(s, x)-\frac{\partial}{\partial s} u_{h}^{(\varepsilon)}(t, y)\right| \leq & 2\left|\frac{\partial}{\partial s} u_{h}^{(\varepsilon)}\right|_{0} \leq N \varepsilon^{-2+\delta} \\
& \leq N \varepsilon^{-2-\delta_{0}+\delta}\left(|t-s|^{\frac{\delta_{0}}{2}}+|x-y|^{\delta_{0}}\right)
\end{aligned}
$$


and (3.9) is proved.

Using similar arguments, Assumption 1 and estimates (3.4)-(3.8), we easily prove that for each $\alpha \in A, i, j=1, \ldots, d$

$$
\begin{aligned}
& {\left[\frac{\partial^{2}}{\partial x_{i} \partial x_{j}} u_{h}^{(\varepsilon)}\right]_{\frac{\delta_{0}}{2}, \delta_{0}}+\left[\frac{\partial}{\partial x_{i}} u_{h}^{(\varepsilon)}\right]_{\frac{\delta_{0}}{2}, \delta_{0}} \leq N \varepsilon^{-2-\delta_{0}+\delta}} \\
& {\left[a_{i j}(\alpha, \cdot, \cdot) \frac{\partial^{2}}{\partial x_{i} \partial x_{j}} u_{h}^{(\varepsilon)}(\cdot, \cdot)\right]_{\frac{\delta_{0}}{2}, \delta_{0}}+\left[b_{i}(\alpha, \cdot, \cdot) \frac{\partial}{\partial x_{i}} u_{h}^{(\varepsilon)}(\cdot, \cdot)\right]_{\frac{\delta_{0}}{2}, \delta_{0}} \leq N \varepsilon^{-2-\delta_{0}+\delta} .}
\end{aligned}
$$

It remains to prove the estimate

$$
\left[\int \nabla_{c(\alpha, \cdot, \cdot, z)}^{2} u_{h}^{(\varepsilon)}(\cdot, \cdot) \Pi(d z)\right]_{\frac{\delta_{0}}{2}, \delta_{0}} \leq N \varepsilon^{-2-\delta_{0}+\delta} .
$$

Obviously,

$$
\begin{aligned}
& \left|\int \nabla_{c(\alpha, s, x, z)}^{2} u_{h}^{(\varepsilon)}(s, x) \Pi(d z)-\int \nabla_{c(\alpha, t, y, z)}^{2} u_{h}^{(\varepsilon)}(t, y) \Pi(d z)\right| \\
& \leq \int\left|\nabla_{c(\alpha, s, x, z)}^{2} u_{h}^{(\varepsilon)}(s, x)-\nabla_{c(\alpha, s, x, z)}^{2} u_{h}^{(\varepsilon)}(t, y)\right| \Pi(d z) \\
& \quad+\int\left|\nabla_{c(\alpha, s, x, z)}^{2} u_{h}^{(\varepsilon)}(t, y)-\nabla_{c(\alpha, t, y, z)}^{2} u_{h}^{(\varepsilon)}(t, y)\right| \Pi(d z) \equiv I_{1}+I_{2} .
\end{aligned}
$$

Using the formula

$$
\nabla_{y}^{2} u_{h}^{(\varepsilon)}(s, x)=\sum_{i, j=1}^{d} \int_{0}^{1}(1-\Theta) \frac{\partial^{2} u_{h}^{(\varepsilon)}}{\partial x_{i} \partial x_{j}}(s, x+\Theta y) d \Theta y_{i} y_{j}
$$

(3.10) and Assumption 1, we have

$$
\begin{gathered}
I_{1} \leq \sum_{i, j=1}^{d} \int \mid \int_{0}^{1}(1-\Theta)\left[\frac{\partial^{2}}{\partial x_{i} \partial x_{j}} u_{h}^{(\varepsilon)}(s, x+\Theta c(\alpha, s, x, z))\right. \\
\left.-\frac{\partial^{2}}{\partial x_{i} \partial x_{j}} u_{h}^{(\varepsilon)}(t, y+\Theta c(\alpha, s, x, z))\right] d \Theta c_{i}(\alpha, s, x, z) c_{j}(\alpha, s, x, z) \mid \Pi(d z) \\
\leq N \max _{i, j=1, \ldots, d}\left[\frac{\partial^{2}}{\partial x_{i} \partial x_{j}} u_{h}^{(\varepsilon)}\right]_{\frac{\delta_{0}}{2}, \delta_{0}}\|c(\alpha, s, x, \cdot)\|_{2, \Pi}^{2} \\
\times\left(|t-s|^{\frac{\delta_{0}}{2}}+|x-y|^{\delta_{0}}\right) \leq N \varepsilon^{-2-\delta_{0}+\delta}\left(|t-s|^{\frac{\delta_{0}}{2}}+|x-y|^{\delta_{0}}\right) .
\end{gathered}
$$

To estimate $I_{2}$, we notice that for each $\xi, \eta \in R^{d}$

$$
\begin{aligned}
& \left|\nabla_{\xi}^{2} u_{h}^{(\varepsilon)}(t, y)-\nabla_{\eta}^{2} u_{h}^{(\varepsilon)}(t, y)\right| \\
& \quad=\left|\sum_{i=1}^{d} \int_{0}^{1}\left[\frac{\partial}{\partial x_{i}} u_{h}^{(\varepsilon)}(t, y+\eta+\Theta(\xi-\eta))-\frac{\partial}{\partial x_{i}} u_{h}^{(\varepsilon)}(t, y)\right] d \Theta\left(\xi_{i}-\eta_{i}\right)\right| \\
& \quad \leq N \max _{i, j=1, \ldots, d}\left|\frac{\partial^{2}}{\partial x_{i} \partial x_{j}} u_{h}^{(\varepsilon)}\right|_{0}(|\xi|+|\eta|)|\xi-\eta| .
\end{aligned}
$$


Therefore, by Hölder's inequality, (3.6) and Assumption 1,

$$
\begin{aligned}
I_{2} & \leq N \varepsilon^{-2+\delta} \int(|c(\alpha, s, x, z)|+|c(\alpha, t, y, z)|)|c(\alpha, s, x, z)-c(\alpha, t, y, z)| \Pi(d z) \\
& \leq N \varepsilon^{-2+\delta}\left(\|c(\alpha, s, x, \cdot)\|_{2, \Pi}+\|c(\alpha, t, y, \cdot)\|_{2, \Pi}\right)\|c(\alpha, s, x, \cdot)-c(\alpha, t, y, \cdot)\|_{2, \Pi} \\
& \leq N \varepsilon^{-2+\delta}\left(|t-s|^{\frac{\delta_{0}}{2}}+|x-y| \wedge 1\right) \leq N \varepsilon^{-2+\delta}\left(|t-s|^{\frac{\delta_{0}}{2}}+|x-y|^{\delta_{0}} .\right.
\end{aligned}
$$

The lemma is proved.

\section{Proof of Main Results}

Proof of Theorem 1. Since $v_{h} \leq v$, it suffices to prove that for each $s \in[0, T]$ and $x \in R^{d}$

$$
v(s, x) \leq v_{h}(s, x)+N h^{\varkappa} .
$$

If $s \in\left[T-h^{2}, T\right]$, then, by Remark 3 , for each $\alpha \in \mathfrak{A}$

$$
\left|v^{\alpha}(s, x)-g(x)\right| \leq \mathbb{E}\left|g\left(X_{T-s}^{\alpha, s, x}\right)-g(x)\right| \leq K \mathbb{E}\left|X_{T-s}^{\alpha, s, x}-x\right|^{\delta} \leq N h^{\delta} .
$$

Thus we have to prove (4.1) for $s \leq T-h^{2}$ assuming without loss of generality that $T \geq h^{2}$. Denote $\varepsilon=h^{\bar{\varkappa} /\left(\delta \delta_{0}\right)}$. Let $\beta=(\alpha, r, \xi) \in \mathfrak{B}_{h}$ and $t \leq h^{2}$. As can be easily seen,

$$
X_{t}^{\beta, s, x}=X_{t}^{\alpha, s+\varepsilon^{2} r, x+\varepsilon \xi}-\varepsilon \xi .
$$

Therefore, by Remark 2 , for each $\beta=(\alpha, r, \xi) \in B, s \in\left[0, S-h^{2}\right]$ and $x \in R^{d}$

$$
u_{h}(s, x) \geq \mathbb{E} u_{h}\left(s+h^{2}, X_{h^{2}}^{\beta, s, x}\right)=\mathbb{E} u_{h}\left(s+h^{2}, X_{h^{2}}^{\alpha, s+\varepsilon^{2} r, x+\varepsilon \xi}-\varepsilon \xi\right)
$$

or for each $\alpha \in A, r \in(-1,0), \xi \in B_{1}, s \leq S-h^{2}+\varepsilon^{2} r$ and $x \in R^{d}$

$$
u_{h}\left(s-\varepsilon^{2} r, x-\varepsilon \xi\right) \geq \mathbb{E} u_{h}\left(s-\varepsilon^{2} r+h^{2}, X_{h^{2}}^{\alpha, s, x}-\varepsilon \xi\right) .
$$

Multiplying the last inequality by the smooth kernel $\zeta$ defined in Section 3 and integrating, we get for each $\alpha \in A, s \leq T-h^{2}$ and $x \in R^{d}$

$$
u_{h}^{(\varepsilon)}(s, x) \geq \mathbb{E} u_{h}^{(\varepsilon)}\left(s+h^{2}, X_{h^{2}}^{\alpha, s, x}\right) .
$$

This inequality, together with Itô's formula, implies

$$
\mathbb{E} \int_{0}^{h^{2}} L^{\alpha} u_{h}^{(\varepsilon)}\left(s+r, X_{r}^{\alpha, s, x}\right) d r \leq 0 .
$$

Hence, by Remark 3 and Lemma 6 , for each $\alpha \in A, s \leq T-h^{2}$ and $x \in R^{d}$

$$
\begin{aligned}
& L^{\alpha} u_{h}^{(\varepsilon)}(s, x) \leq \frac{1}{h^{2}} \mathbb{E} \int_{0}^{h^{2}}\left[L^{\alpha} u_{h}^{(\varepsilon)}(s, x)-L^{\alpha} u_{h}^{(\varepsilon)}\left(s+r, X_{r}^{\alpha, s, x}\right)\right] d r \\
& \quad \leq\left[L^{\alpha} u_{h}^{(\varepsilon)}\right]_{\frac{\delta_{0}}{2}, \delta_{0}} \frac{1}{h^{2}} \int_{0}^{h^{2}}\left(r^{\frac{\delta_{0}}{2}}+\mathbb{E}\left|X_{r}^{\alpha, s, x}-x\right|^{\delta_{0}}\right) d r \leq N \varepsilon^{-2-\delta_{0}+\delta} h^{\delta_{0}} .
\end{aligned}
$$


Therefore, by Itô's formula, for each $\alpha \in \mathfrak{A}, s \leq T-h^{2}$ and $x \in R^{d}$

$$
\begin{aligned}
& \mathbb{E} u_{h}^{(\varepsilon)}\left(T-h^{2}, X_{T-h^{2}-s}^{\alpha, s, x}\right)-u_{h}^{(\varepsilon)}(s, x) \\
& \quad=\mathbb{E} \int_{0}^{T-h^{2}-s} L^{\alpha_{t}} u_{h}^{(\varepsilon)}\left(s+t, X_{t}^{\alpha, s, x}\right) d t \leq N \varepsilon^{-2-\delta_{0}+\delta} h^{\delta_{0}} .
\end{aligned}
$$

Since, by Lemma 5 ,

$$
\begin{aligned}
\left|u_{h}^{(\varepsilon)}(s, x)-u_{h}(s, x)\right| & \leq \int_{R^{d+1}}\left|u_{h}\left(s-\varepsilon^{2} r, x-\varepsilon \xi\right)-u_{h}(s, x)\right| \zeta(r, \xi) d r d \xi \\
& \leq N\left(h^{\delta \delta_{0}}+\varepsilon^{\delta}\right) \leq N \varepsilon^{\delta},
\end{aligned}
$$

we have

$$
\mathbb{E} u_{h}\left(T-h^{2}, X_{T-h^{2}-s}^{\alpha, s, x}\right) \leq u_{h}(s, x)+N\left(\varepsilon^{\delta}+\varepsilon^{-2-\delta_{0}+\delta} h^{\delta_{0}}\right) .
$$

Furthermore, by Lemma 5,

$$
\begin{aligned}
\left|u_{h}\left(T-h^{2}, x\right)-g(x)\right| & =\left|u_{h}\left(T-h^{2}, x\right)-u_{h}(S, x)\right| \\
& \leq N\left(\left(\varepsilon^{2}+h^{2}\right)^{\frac{\delta}{2}}+h^{\delta \delta_{0}}\right) \leq N \varepsilon^{\delta} .
\end{aligned}
$$

Hence

$$
\mathbb{E} g\left(X_{T-h^{2}-s}^{\alpha, s, x}\right) \leq u_{h}(s, x)+N\left(\varepsilon^{\delta}+\varepsilon^{-2-\delta_{0}+\delta} h^{\delta_{0}}\right) .
$$

and, by Remark 3 and Lemma 4 ,

$$
\begin{aligned}
v^{\alpha}(s, x) & =\mathbb{E} g\left(X_{T-s}^{\alpha, s, x}\right) \leq \mathbb{E}\left|g\left(X_{T-s}^{\alpha, s, x}\right)-g\left(X_{T-s-h^{2}}^{\alpha, s, x}\right)\right|+\mathbb{E} g\left(X_{T-s-h^{2}}^{\alpha, s, x}\right) \\
& \leq K \mathbb{E} \mid X_{T-s}^{\alpha, s, x}-X_{T-s-h^{2}}^{\alpha, s, x}+\mathbb{E} g\left(X_{T-s-h^{2}}^{\alpha, s, x}\right) \\
& \leq u_{h}(s, x)+N\left(h^{\delta}+\varepsilon^{\delta}+\varepsilon^{-2-\delta_{0}+\delta} h^{\delta_{0}}\right) \\
& \leq v_{h}(s, x)+N\left(\varepsilon^{\delta_{0} \delta} \varepsilon^{-2-\delta_{0}+\delta} h^{\delta_{0}}\right) .
\end{aligned}
$$

The theorem is proved.

Proof of Theorem 2. Fix $h \in(0,1)$ and for $\alpha \in \mathfrak{B}_{h}$ define the process $\bar{x}_{t}=$ $\bar{x}_{t}^{\alpha, s, x}(h)$ recursively by

$$
\begin{aligned}
\bar{x}_{0}= & x \\
\bar{x}_{t}= & \bar{x}_{n h^{2}}+\sigma\left(\alpha_{n h^{2}}, s+n h^{2}, \bar{x}_{n h^{2}}\right)\left(W_{t}-W_{n h^{2}}\right) \\
& +b\left(\alpha_{n h^{2}}, s+n h^{2}, \bar{x}_{n h^{2}}\right)\left(t-n h^{2}\right) \\
& +\int_{n h^{2}}^{t} \int_{R^{m}} c\left(\alpha_{n h^{2}}, s+n h^{2}, \bar{x}_{n h^{2}}, z\right) q(d r, d z)
\end{aligned}
$$

for $n h^{2} \leq t \leq(n+1) h^{2}$. It is easy to see that $\bar{x}_{t}^{\alpha, s, x}(h)$ satisfies the equation

$$
\begin{aligned}
\bar{x}_{t}= & x+\int_{0}^{t} \sigma\left(\alpha_{r}, s+\varkappa_{h}(r), \bar{x}_{\varkappa_{h}(r)}\right) d W_{r}+\int_{0}^{t} b\left(\alpha_{r}, s+\varkappa_{h}(r), \bar{x}_{\varkappa_{h}(r)}\right) d r \\
& +\int_{0}^{t} \int_{R^{m}} c\left(\alpha_{r}, s+\varkappa_{h}(r), \bar{x}_{\varkappa_{h}(r)}, z\right) q(d r, d z),
\end{aligned}
$$


where $\varkappa_{h}(t)=h^{2}\left[\frac{t}{h^{2}}\right]$. Let

$$
\bar{v}_{h}^{\alpha}(s, x)=\mathbb{E} g\left(\bar{x}_{\varkappa_{h}(T-s)}^{\alpha, s, x}(h)\right) .
$$

Notice that the function $\bar{v}_{h}$ defined in Section 2 satisfies the dynamic programming equation for the problem of maximizing $\bar{v}_{h}^{\alpha}(s, x)$ over $\alpha \in \mathfrak{A}_{h}$. Therefore,

$$
\bar{v}_{h}(s, x)=\sup _{\alpha \in \mathfrak{A}_{h}} \bar{v}_{h}^{\alpha}(s, x) .
$$

Rewrite (4.2) as

$$
\bar{x}_{t}=x+\int_{0}^{t} \bar{\sigma}_{r}\left(\bar{x}_{r}\right) d W_{r}+\int_{0}^{t} \bar{b}_{r}\left(\bar{x}_{r}\right) d r+\int_{0}^{t} \int_{R^{m}} \bar{c}_{r}\left(\bar{x}_{r}, z\right) q(d r, d z),
$$

where

$$
\bar{\sigma}_{t}(y)=\sigma\left(\alpha_{t}, s+\varkappa_{h}(t), y+\bar{x}_{\varkappa_{h}(t)}^{\alpha, s, x}(h)-\bar{x}_{t}^{\alpha, s, x}(h)\right)
$$

and similarly are defined $\bar{b}_{t}(y)$ and $\bar{c}_{t}(y, z)$. Then, by Lemma 2 , for each $\alpha \in \mathfrak{A}_{h}$, $s \in[0, T]$ and $x \in R^{d}$

$$
\begin{aligned}
\mathbb{E} \sup _{t \leq T} & \left|\bar{x}_{t}^{\alpha, s, x}-X_{t}^{\alpha, s, x}\right|^{2} \leq N \mathbb{E} \int_{0}^{T}\left(\left\|\sigma\left(\alpha_{t}, s+t, X_{t}^{\alpha, s, x}\right)-\bar{\sigma}_{t}\left(X_{t}^{\alpha, s, x}\right)\right\|^{2}\right. \\
& +\left|b\left(\alpha_{t}, s+t, X_{t}^{\alpha, s, x}\right)-\bar{b}_{t}\left(X_{t}^{\alpha, s, x}\right)\right|^{2} \\
& \left.+\left\|c\left(\alpha_{t}, s+t, X_{t}^{\alpha, s, x}, \cdot\right)-\bar{c}_{t}\left(X_{t}^{\alpha, s, x}, \cdot\right)\right\|_{2, \Pi}^{2}\right) d t \\
\leq & N \int_{0}^{t}\left(h^{2 \delta_{0}}+\mathbb{E}\left|\bar{x}_{\varkappa, s(t)}^{\alpha, s, x}(h)-\bar{x}_{t}^{\alpha, s, x}(h)\right|^{2}\right) d t \leq N h^{2 \delta_{0}} .
\end{aligned}
$$

This estimate and Remark 3 imply that for each $\alpha \in \mathfrak{A}_{h}, s \in[0, T]$ and $x \in R^{d}$

$$
\begin{aligned}
\left|v_{h}^{\alpha}(s, x)-\bar{v}_{h}^{\alpha}(s, x)\right| & \leq \mathbb{E}\left|g\left(X_{(T-s)}^{\alpha, s, x}\right)-g\left(\bar{x}_{\varkappa_{h}(T-s)}^{\alpha, s, x}(h)\right)\right| \\
& \leq K \mathbb{E}\left|X_{(T-s)}^{\alpha, s, x}-\bar{x}_{\varkappa_{h}(T-s)}^{\alpha, s, x}(h)\right|^{\delta} \leq N h^{\delta \delta_{0}} .
\end{aligned}
$$

Hence, for each $s \in[0, T]$ and $x \in R^{d}$

$$
\left|v_{h}(s, x)-\bar{v}_{h}(s, x)\right| \leq N h^{\delta \delta_{0}}
$$

what, together with Theorem 1, implies the assertion of the theorem.

\section{References}

[1] N.V. Krylov. Controlled Diffusion Processes. Springer, Berlin, 1980.

[2] N.V. Krylov. Approximating value functions for controlled degenerate diffusion processes by using piece-wise constant policies. Electron. J. Probab., 4:1-19, 1999.

[3] N.V. Krylov and H. Pragarauskas. Traditional derivation of Bellman equation for general controlled stochastic processes. Lith. Math. J., 2(21):146-152, 1981.

[4] H. Pragarauskas. Bellman's equation in a lattice of measures for general controlled stochastic processes. I. Lith. Math. J., 21(4):353-363, 1981. Doi:10.1007/BF00969856. 
[5] H. Pragarauskas. Bellman's equation in a lattice of measures for general controlled stochastic processes. II. Lith. Math. J., 22(1):68-73, 1982. Doi:10.1007/BF00967929.

[6] H. Pragarauskas. Approximation of controlled solution of Ito's equation by controlled Markov chains. Lith. Math. J., 1(23):98-108, 1983. Doi:10.1007/BF00968597. 